\title{
Erratum: Neural network potential for Al-Mg-Si alloys [Phys. Rev. Materials 1, 053604 (2017)]
}

\author{
Ryo Kobayashi, Daniele Giofré, Till Junge, Michele Ceriotti, and William A. Curtin
}

(Received 13 November 2017; published 29 November 2017)

DOI: 10.1103/PhysRevMaterials.1.069901

The values of $C_{12}$ in Table I of the original article are wrong and the corrected table is shown below. The $C_{12}$ values shown in the original article are $C_{44}$ values and the $C_{12}$ values are corrected in this Table I. This correction does not affect the discussion and conclusion of the article.

TABLE I. Pure Al bulk properties from experiments or $a b$ initio calculations and as computed using the present NN potential and two MEAM potentials (Jelinek and Kim). The structures relevant to these properties are in the training data.

\begin{tabular}{lllll}
\hline \hline $\mathrm{Al}$ & \multicolumn{1}{c}{ Expt./ab initio } & $\mathrm{NN}$ & \multicolumn{1}{c}{ Jelinek } & Kim \\
\hline$a(\AA)$ & $4.05[31], 4.045$ & 4.045 & 4.048 & 4.047 \\
$E_{\mathrm{c}}(\mathrm{eV})$ & $3.39[32], 3.057$ & 3.057 & 3.353 & 3.360 \\
$B(\mathrm{GPa})$ & $72.2[32], 79.1$ & 76.9 & 78.4 & 79.0 \\
$C_{11}(\mathrm{GPa})$ & $114.3[32], 106.1[19]$ & 109.9 & 111.1 & 113.8 \\
$C_{12}(\mathrm{GPa})$ & $61.9[32], 55.9[19]$ & 55.5 & 60.9 & 61.4 \\
$C_{44}(\mathrm{GPa})$ & $31.6[32], 31.9[19]$ & 31.6 & 28.6 & 31.4 \\
$\gamma_{\mathrm{sf}}\left(\mathrm{mJ} / \mathrm{m}^{2}\right)$ & $135-166[32,33], 122-164[29,34-38], 125.8$ & 116.4 & 141.9 & 147.1 \\
$\gamma_{\text {us }}\left(\mathrm{mJ} / \mathrm{m}^{2}\right)$ & $224[37,38], 166.2$ & 156.7 & 280.9 & 236.2 \\
$\gamma_{(111)}\left(\mathrm{mJ} / \mathrm{m}^{2}\right)$ & $710[38], 720.2$ & 742.9 & 716.1 & 516.3 \\
$\gamma_{(001)}\left(\mathrm{mJ} / \mathrm{m}^{2}\right)$ & 873.8 & 878.0 & 1071.6 & 743.9 \\
$\gamma_{(110)}\left(\mathrm{mJ} / \mathrm{m}^{2}\right)$ & 927.7 & 945.1 & 1104.8 & 820.9 \\
$\alpha\left(10^{-6} / \mathrm{K}\right)$ & $23.6-25.4[39]$ & 23.3 & 14.4 & \\
\hline \hline
\end{tabular}

